\title{
openheart Effects of adding ivabradine to usual care in patients with angina pectoris: a systematic review of randomised clinical trials with meta-analysis and Trial Sequential Analysis
}

Mathias Maagaard (D) , ${ }^{1}$ Emil Eik Nielsen (i) , ${ }^{2}$ Naqash Javaid Sethi, ${ }^{1}$ Liang Ning, ${ }^{3,4}$ Si-hong Yang, ${ }^{4}$ Christian Gluud, ${ }^{5}$ Janus Christian Jakobsen ${ }^{1,6}$

\begin{abstract}
- Additional material is published online only. To view, please visit the journal online (http://dx.doi.org/10.1136/ openhrt-2020-001288).
\end{abstract}

To cite: Maagaard $\mathrm{M}$, Nielsen EE, Sethi NJ, et al. Effects of adding ivabradine to usual care in patients with angina pectoris: a systematic review of randomised clinical trials with meta-analysis and Trial Sequential Analysis. Open Heart 2020;7:e001288.

doi:10.1136/

openhrt-2020-001288

Received 16 March 2020

Revised 18 June 2020

Accepted 19 August 2020

Check for updates

(C) Author(s) (or their employer(s)) 2020. Re-use permitted under CC BY-NC. No commercial re-use. See rights and permissions. Published by BMJ.

For numbered affiliations see end of article.

Correspondence to Dr Mathias Maagaard; mathias. maagaard@ctu.dk

\section{ABSTRACT}

Objective To determine the impact of ivabradine on outcomes important to patients with angina pectoris caused by coronary artery disease.

Methods We conducted a systematic review. We included randomised clinical trials comparing ivabradine versus placebo or no intervention for patients with angina pectoris due to coronary artery disease published prior to June 2020. We used Preferred Reporting Items for Systematic Reviews and Meta-Analyses guidelines, Cochrane methodology, Trial Sequential Analysis, Grading of Recommendations Assessment, Development, and Evaluation, and our eight-step procedure. Primary outcomes were all-cause mortality, serious adverse events and quality of life.

Results We included 47 randomised clinical trials enrolling 35797 participants. All trials and outcomes were at high risk of bias. Ivabradine compared with control did not have effects when assessing all-cause mortality (risk ratio [RR] 1.04; $95 \% \mathrm{Cl} 0.96$ to 1.13 ), quality of life (standardised mean differences $-0.05 ; 95 \% \mathrm{Cl}-0.11$ to $0.01)$, cardiovascular mortality (RR $1.07 ; 95 \% \mathrm{Cl} 0.97$ to 1.18) and myocardial infarction (RR $1.03 ; 95 \% \mathrm{Cl} 0.91$ to 1.16). Ivabradine seemed to increase the risk of serious adverse events after removal of outliers (RR $1.07 ; 95 \% \mathrm{Cl}$ 1.03 to 1.11) as well as the following adverse events classified as serious: bradycardia, prolonged QT interval, photopsia, atrial fibrillation and hypertension. Ivabradine also increased the risk of non-serious adverse events (RR 1.13; 95\% $\mathrm{Cl} 1.11$ to 1.16). Ivabradine might have a statistically significant effect when assessing angina frequency (mean difference (MD) 2.06; $95 \% \mathrm{Cl} 0.82$ to 3.30 ) and stability (MD 1.48; $95 \% \mathrm{Cl} 0.07$ to 2.89), but the effect sizes seemed minimal and possibly without any relevance to patients, and we identified several methodological limitations, questioning the validity of these results.

Conclusion Our findings do not support that ivabradine offers significant benefits on patient important outcomes, but rather seems to increase the risk of serious adverse events such as atrial fibrillation and non-serious adverse events. Based on current evidence, guidelines need reassessment and the use of ivabradine for angina pectoris should be reconsidered.

\section{Key questions}

What is already known about this subject?

- Ivabradine is recommended in European Society of Cardiology guidelines on chronic coronary syndromes. In the 'ivabradine for patients with stable coronary artery disease and left-ventricular systolic dysfunction' (BEAUTIFUL) trial, ivabradine seemed to reduce the incidence of coronary artery disease outcomes in patients with a heart rate of 70 beats $/ \mathrm{min}$ or higher. In the 'ivabradine in stable coronary artery disease without clinical heart failure' (SIGNIFY) trial, ivabradine did not improve clinical outcomes. Previous studies have shown a beneficial effect of ivabradine on angina pectoris symptoms. To our knowledge, no previous systematic review has assessed the effects of ivabradine compared with placebo or no intervention, searching all relevant databases, and considering both risks of systematic errors and random errors.

What does this study add?

- Our findings do not support that ivabradine offers significant benefits on patient important outcomes, but rather seems to increase the risk of serious adverse events such as atrial fibrillation and nonserious adverse events.

How might this impact on clinical practice?

- Based on current evidence, guidelines need reassessment and the use of ivabradine for angina pectoris should be reconsidered.

PROSPERO registration number CRD42018112082.

\section{INTRODUCTION}

Cardiovascular diseases accounts for 30\% of all deaths worldwide. ${ }^{1}$ Ischaemic heart disease is associated with an increased risk of mortality and morbidity with an estimated global prevalence over 110 million in $2015 .^{2}$ 
Coronary artery disease is characterised by recurrent episodes of a mismatch between myocardial oxygen supply and demand, resulting in myocardial ischaemia and chest discomfort known as angina pectoris. ${ }^{3}$

Ivabradine is a selective sinus node inhibitor, exerting its effect by decreasing heart rate, thereby decreasing myocardial oxygen demand and increasing myocardial oxygen supply. Theoretically, ivabradine might be an effective intervention for angina pectoris caused by coronary artery disease..$^{4-7}$ To our knowledge, no previous systematic review has assessed the effects of ivabradine compared with usual care (ie, placebo or no intervention) for angina pectoris, searching all relevant databases, and considering both risk of systematic errors and random errors. ${ }^{8-12}$

\section{METHODS}

Our methodology is described in detail in our protocol published prior to conducting the literature search. ${ }^{13} 14$

In short, we carried out this systematic review following the recommendations of the Preferred Reporting Items for Systematic Reviews and Meta-Analyses guidelines. ${ }^{12}$ We included all trials comparing ivabradine versus placebo or no intervention (ie, usual care plus ivabradine vs usual care alone) for participants with angina pectoris. We searched various databases for randomised clinical trials published prior to June 2020, see 'online supplemental 1' for a detailed list of databases. Out search strategy is shown in 'online supplemental 2'. We included randomised clinical trials regardless of trial design, setting, publication status, year, language and reporting of outcomes. Two authors (MM and EEN) independently screened for randomised clinical trials in all non-Chinese databases. The Chinese databases were independently screened by two other authors (LN and SY). Three authors independently extracted data and assessed the risks of bias in the non-Chinese trials (MM all included trials, EEN and NS half each) and two other authors independently extracted data and assessed the risk of bias in the Chinese trials (LN and SY). We attempted to contact trial authors if data were unclear or missing. Disagreements were resolved through discussion or by consulting another author (JCJ). ${ }^{14}$

We assessed three primary outcomes: all-cause mortality, serious adverse events and quality of life. We also assessed three secondary outcomes and eight exploratory outcomes. ${ }^{14}$ For all outcomes, we used the trial results reported at maximal follow-up. We chose to assess quality of life using standardised mean differences (SMD), due to the trials reporting on quality of life using different scales. As a 'rule of thumb', an effect below 0.4 is a small effect, 0.4 to 0.7 is a moderate effect and above 0.7 is a large effect. ${ }^{15}$

We predefined several subgroup analyses for the primary outcomes, and we conducted sensitivity analyses when assessing both primary and secondary outcomes (see the Results section for sensitivity analyses and see 'online supplemental material' for subgroup analyses). ${ }^{14}$

\section{Assessment of statistical and clinical significance}

We performed all meta-analyses using Review Manager V.5.3. ${ }^{16}$ To control for random errors, we used Trial Sequential Analysis and adjusted the threshold for statistical significance as suggested by Jakobsen and colleagues. ${ }^{81017}$ We used three primary outcomes and therefore considered a $\mathrm{p}$ value of 0.025 as the threshold for statistical significance. ${ }^{10}$ When analysing secondary and exploratory outcomes, we considered a $\mathrm{p}$ value of 0.05 as the threshold for statistical significance. ${ }^{10}$ We reported the Trial Sequential Analysis-adjusted confidence intervals and if the cumulative Z-curves crossed any of the Trial Sequential Analysis boundaries (benefit, harm or futility). In order to control the risk of random error when assessing the individual serious and nonserious adverse events, we further adjusted our thresholds for statistical significance according to the large number of comparisons (see 'Serious adverse events' and 'Non-serious adverse events'). Hence, we post-hoc considered a $p$ value of 0.001 as threshold for statistical significance when analysing individual serious and nonserious adverse events.

We used a 'best-worst case' and a 'worst-best case' analysis to assess the impact of missing data. ${ }^{15}$ We used GRADE to assess the certainty of evidence. ${ }^{1819}$

\section{RESULTS}

Our literature search identified 4452 records from databases. We also identified 11 unpublished trials on the trial platform of the company, Servier, that developed ivabradine. ${ }^{20}$ After removing duplicates, 3058 records remained. We excluded 2846 records based on title or abstract. We excluded another 165 records based on full text, see 'online supplemental 3'. We included 42 randomised clinical trials from databases. We included five unpublished, randomised clinical trials from Servier. ${ }^{21-25}$ Therefore, we included a total of 47 randomised clinical trials randomising 35 797 participants. ${ }^{21-67}$ Twenty trials compared ivabradine with placebo ${ }^{21-2528-32} 34363940444650545558$ and 27 trials compared ivabradine with 'no intervention'. Of the 16 trials comparing ivabradine with 'no intervention', 14 trials used guideline-based therapy in both trial groups ${ }^{2641} 424547566062-68$ and 13 trials used various cointerventions other than guideline-based therapy in both trial groups (12 trials used specific betablockers $^{27} 3538434851-5357596169$ and one used a calciumchannel blocker). ${ }^{33}$ For baseline characteristics, see table 1. For all primary and secondary outcomes, we chose to analyse data using fixed-effect meta-analysis due to two trials accounting for more than $97 \%$ of weight (see paragraph below)..$^{10} 3254$

The two largest trials, the SIGNIFY trial and the BEAUTIFUL trial, contributed with more than $97 \%$ of weight 

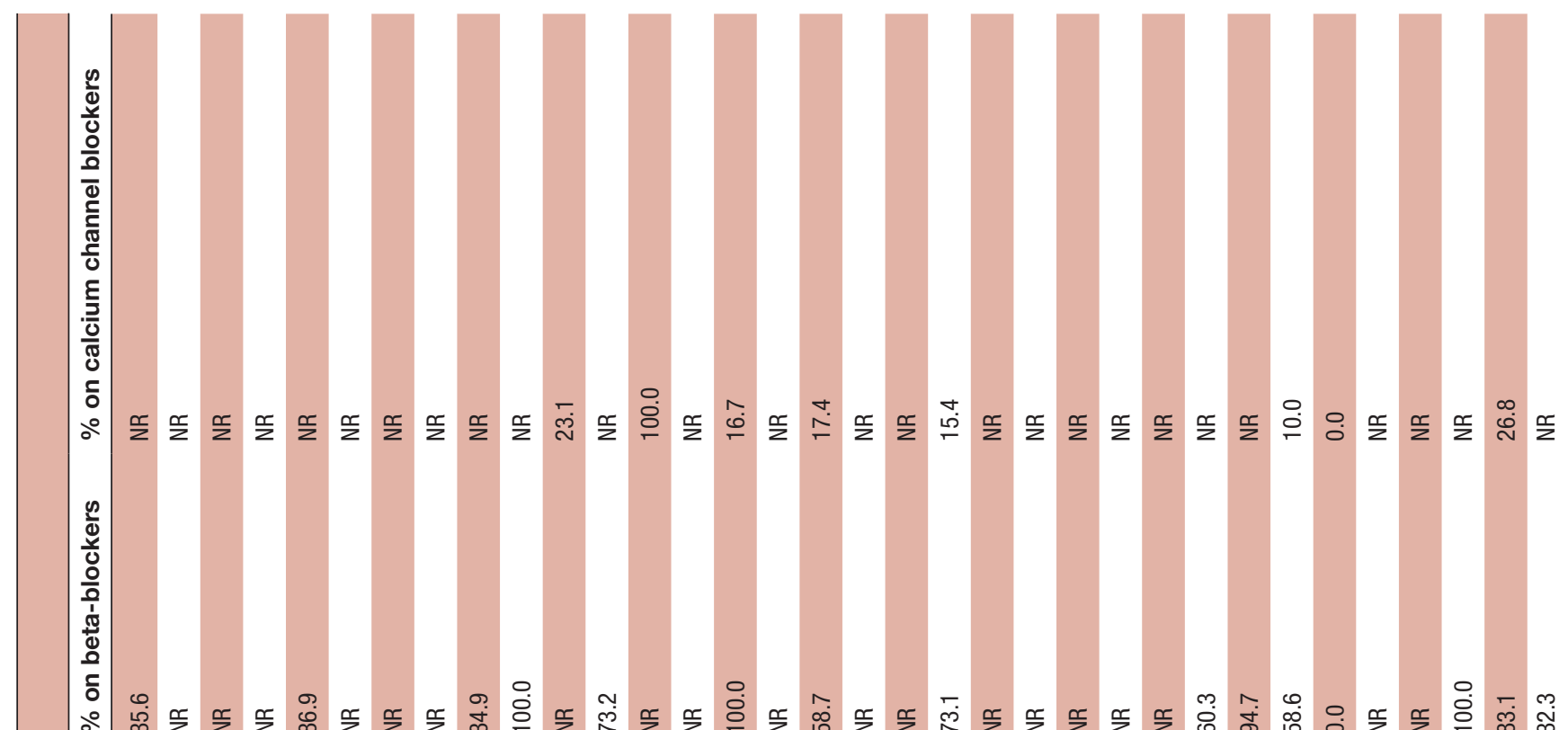

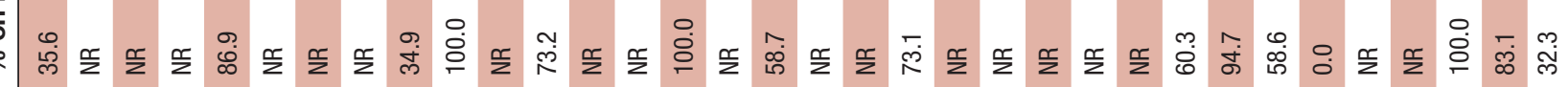

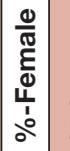

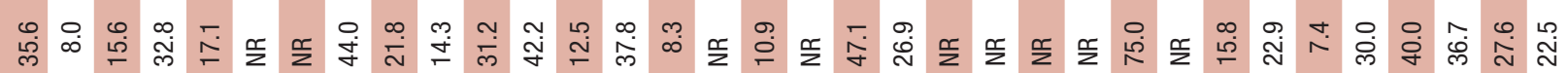

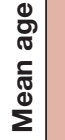

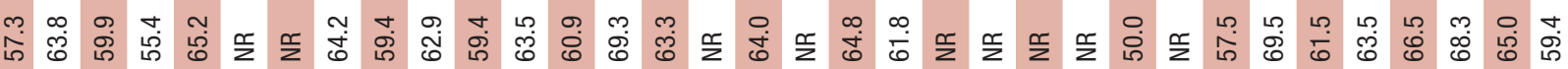

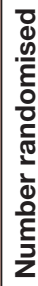

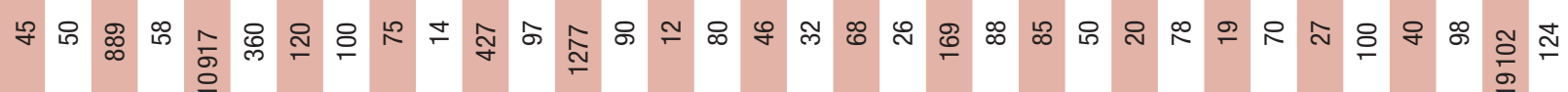

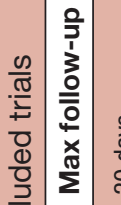

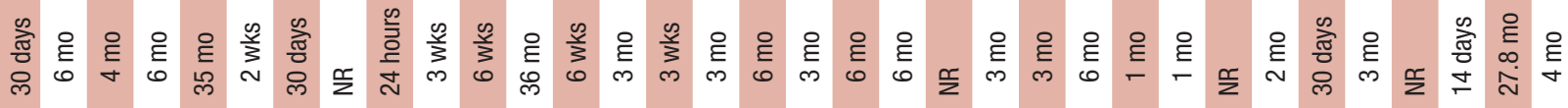

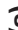

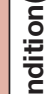

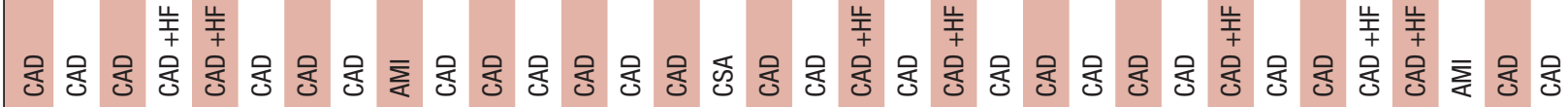

㐫

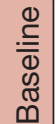

ஆ 융

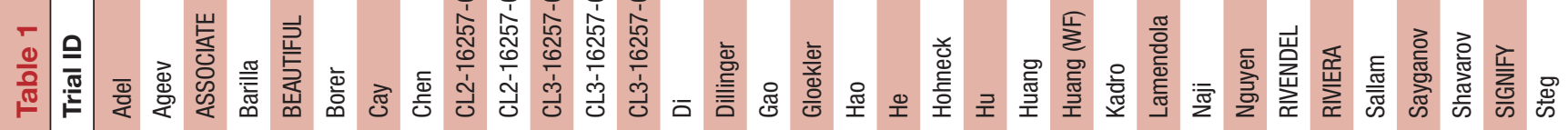




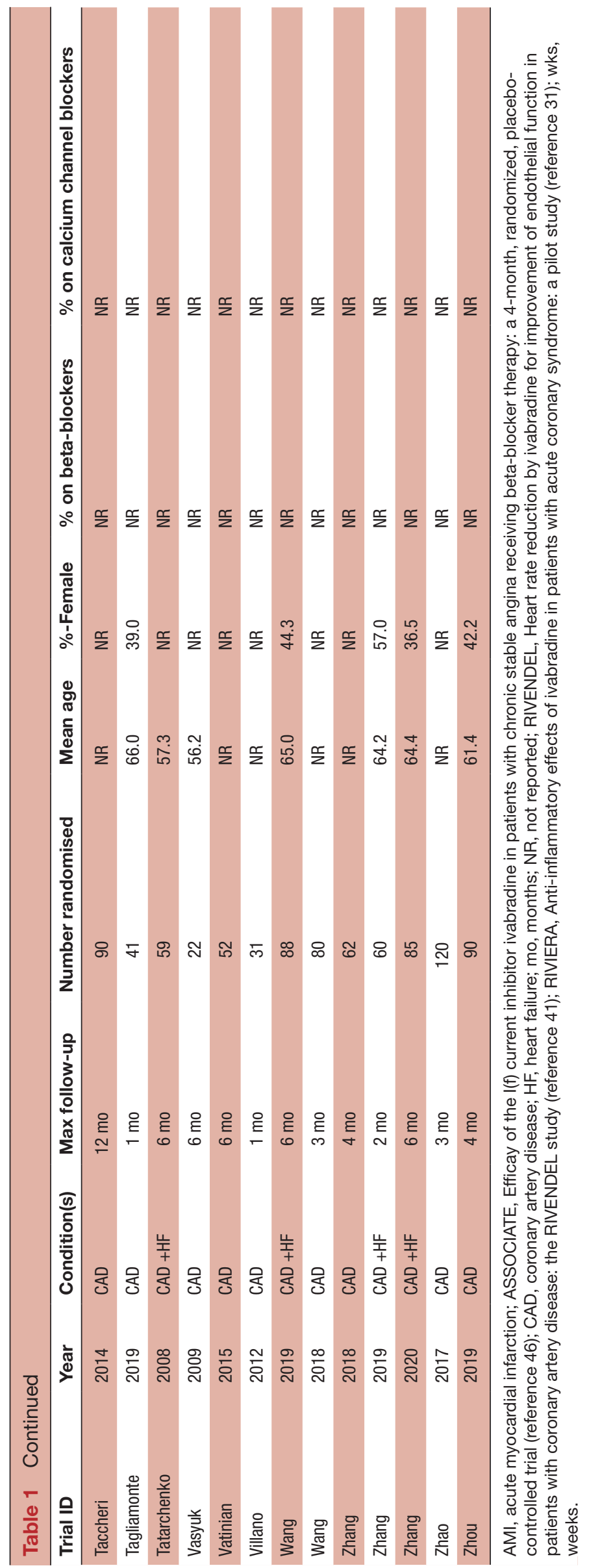


in all primary and secondary outcome meta-analyses. We identified several methodological limitations regarding these two trials. First, both trials were not prospectively registered before randomisation began. ${ }^{32}{ }^{54}$ The BEAUTIFUL trial randomised the first participant in January 2005 and was first registered with ClinicalTrials.gov in September 2005 and sent their rationale article for peer review in November 2005..$^{54} 71$ The SIGNIFY trial randomised the first participant in October 2009 and was first registered with Clinicaltrials.gov in May 2015 and first sent their rationale article for peer review in April 2013. ${ }^{32} 7273$ Therefore, it was not documented that the methodology, including outcomes and participating centres, was predefined before randomisation began. This is especially problematic when assessing composite outcomes consisting of individual components with very different degrees of severity (ie, in the SIGNIFY trial, the primary composite outcome was death from cardiovascular causes or non-fatal myocardial infarction. In the BEAUTIFUL trial, the primary composite outcome was cardiovascular death, admission to hospital for acute myocardial infarction, or admission to hospital for new onset or worsening heat failure). There is a high risk of selective outcome reporting bias, if the composite outcomes are not clearly predefined before randomisation begins. ${ }^{15}$ Furthermore, during the course of the BEAUTIFUL trial, the investigators incorporated a subgroup analysis on participants with a baseline heart rate at or above 70 beats/min (one of the inclusion criteria in the SIGNIFY trial). ${ }^{32}{ }^{54}$ However, there is no documentation for this subgroup analysis being prespecified prior to initiation of the BEAUTIFUL trial. ${ }^{54} 7071$ Second, in the SIGNIFY trial assessing quality of life and angina pectoris, 13871 (72.6\%) of the 19102 participants included in the main study were not included in the analysis. ${ }^{3274}$ It was briefly described in the publication that the reasons were either that some countries did not have a translation of the quality of life scale or it was due to 'lack of consent' ${ }^{74}$ Third, for serious and non-serious adverse events, there were considerable discrepancies between the data reported in the publication of the SIGNIFY trial as compared with the raw data reported on ClinicalTrials. gov, see 'online supplemental 11' ${ }^{32} 73$ Fourth, both the SIGNIFY trial and the BEAUTIFUL trial, as well as all other included trials, were at high risk of bias. Therefore, there is a risk that our results overestimate beneficial effects and underestimate harmful effects. ${ }^{75-80}$

We have contacted the trial authors and the company that produced ivabradine, Servier, but we have not received additional information regarding some of these issues.

See 'online supplemental 4' for a detailed description of the bias risk assessment.

\section{All-cause mortality}

Fifteen trials randomising 33427 participants reported on all-cause mortality. Meta-analysis showed no evidence of a difference between ivabradine and control (RR
$1.04 ; 95 \%$ CI 0.96 to $1.13 ; \mathrm{p}=0.35$; moderate certainty of evidence). Visual inspection of the forest plot and $\mathrm{I}^{2}$-statistics $\left(\mathrm{I}^{2}=0 \%\right)$ indicated no heterogeneity. Trial Sequential Analysis showed that we had enough information to reject that ivabradine versus control reduced the risk of all-cause mortality by $15 \%$ or more (RR $1.03 ; 95 \%$ CI 0.88 to $1.20 ; \mathrm{p}=0.46 ; \mathrm{I}^{2}=0 \% ; \mathrm{D}^{2}=0 \%$ ). This outcome result was assessed at high risk of bias. Incomplete outcome data alone did not seem to have the potential to influence the results. See 'Summary of findingstable' and 'online supplemental 5'.

\section{Serious adverse events}

Eighteen trials randomising 33514 participants reported on serious adverse events. Meta-analysis showed no evidence of a difference between ivabradine and control (RR $1.01 ; 95 \%$ CI 0.98 to $1.04 ; \mathrm{p}=0.56$; moderate certainty of evidence). Visual inspection of the forest plot and $\mathrm{I}^{2}$ statistics $\left(\mathrm{I}^{2}=55 \%\right)$ indicated substantial heterogeneity which could be resolved by removing the BEAUTIFUL trial and the trial by Taccheri $e t$ al from the analysis (RR $1.06 ; 95 \%$ CI 1.02 to $1.10 ; \mathrm{p}=0.001 ; \mathrm{I}^{2}=0 \%$ ). Trial Sequential Analysis, after removing outliers, showed that we had enough information to reject that ivabradine decreased the risk of serious adverse events by $15 \%$ or more (RR 1.06; $95 \%$ CI 1.00 to $\left.1.13 ; \mathrm{p}=0.0014 ; \mathrm{I}^{2}=0 \% ; \mathrm{D}^{2}=0 \%\right)$. This outcome result was assessed at high risk of bias. Incomplete outcome data alone did not seem to have the potential to influence the results. See 'Summary of findingstable' and 'online supplemental 6'.

\section{Individual serious adverse events}

The trials reported on 1749 different serious adverse events, where the SIGNIFY and the BEAUTIFUL trials reported on the majority of those. To limit problems with multiplicity and type 1 errors caused by the unexpected large number of individual serious adverse events, we post-hoc adjusted the threshold for statistical significance to 0.001 when assessing individual serious adverse events. ${ }^{10}$

Ivabradine increased the risk of the following adverse events classified as serious by the trialists: bradycardia (event classified as 'bradycardia' (RR 4.53; 95\% CI 2.99 to $6.87 ; \mathrm{p}<0.0001$; two trials); event classified as 'heart rate decreased' (RR 8.22; 95\% CI 3.85 to 17.54; $\mathrm{p}<0.0001$; two trials); event classified as 'sinus bradycardia' (RR 6.86; 95\% CI 3.11 to 15.15; $\mathrm{p}<0.0001$; one trial)); prolonged QT interval (RR 3.21; $95 \%$ CI 1.90 to 5.40; $<<0.0001$; two trials); photopsia (RR 9.34; $95 \%$ CI 2.84 to $30.71 ; \mathrm{p}=0.0002$; one trial); atrial fibrillation ( $\mathrm{RR}$ 1.26 ; $95 \%$ CI 1.10 to $1.44 ; \mathrm{p}=0.0008$; three trials); and hypertension (RR 1.42; 95\% CI 1.15 to 1.75 ; $\mathrm{p}=0.001$; one trial).

Ivabradine did not seem to decrease the risk of any individual serious adverse events at the threshold of statistical significance of 0.001 . 


\section{Quality of life}

Four trials randomising 4377 participants reported on quality of life analysed as a continuous outcome. One trial used EuroQoL score (0-100 points), ${ }^{50}$ one trial used the Kansas City Cardiomyopathy Questionnaire, ${ }^{59}$ one trial used Minnesota Living with Heart Failure Questionnaire $^{60}$ and the SIGNIFY trial reported on quality of life using the Seattle Angina Questionnaire (which does not result in a combined end-score) and a generic visual analogue scale (0-100 points). ${ }^{32}$ Meta-analysis showed evidence of a beneficial effect of ivabradine (SMD $-0.08 ; 95 \%$ CI -0.14 to $-0.02 ; \mathrm{p}=0.009$; low certainty of evidence). Visual inspection of the forest plot and $\mathrm{I}^{2}$ statistics $\left(I^{2}=97 \%\right)$ indicated substantial heterogeneity, which could be resolved by removing the trials by Sallam et $a \bar{l}^{\tilde{9}}$ and Tatarchenko et $a l^{60}$ (SMD $-0.05 ; 95 \% \mathrm{CI}-0.11$ to $0.01 ; \mathrm{p}=0.09 ; \mathrm{I}^{2}=17 \%$ ). It was not possible to conduct Trial Sequential Analysis using SMD. ${ }^{81}$ This outcome result was assessed as at high risk of bias. We assessed the risk of incomplete outcome data bias to be substantial since $70 \%$ of the participants in the SIGNIFY trial were excluded from the analysis of quality of life, see first section of 'Results'. See 'Summary of findings-table' and 'online supplemental 7'.

\section{Cardiovascular mortality}

Eight trials randomising 32193 participants reported on cardiovascular mortality. Meta-analysis showed no evidence of a difference between ivabradine and control (RR 1.06; 95\% CI 0.96 to 1.17 ; $\mathrm{p}=0.22$; moderate certainty of evidence). Visual inspection of the forest plot and $\mathrm{I}^{2}$-statistics $\left(\mathrm{I}^{2}=14 \%\right)$ indicated low heterogeneity. Trial Sequential Analysis showed that we had enough information to reject that ivabradine versus control reduced the risk of cardiovascular mortality by $15 \%$ or more (RR $1.066 ; 95 \%$ CI 0.95 to $1.18 ; \mathrm{p}=0.22$; $\left.\mathrm{I}^{2}=14 \% ; \mathrm{D}^{2}=53 \%\right)$. This outcome result was assessed at high risk of bias. Incomplete outcome data alone did not seem to have the potential to influence the results. See 'Summary of findings-table' and 'online supplemental 8'.

\section{Myocardial infarction}

Five trials randomising 31810 participants reported on myocardial infarction. Meta-analysis showed no evidence of a difference between ivabradine and control (RR 1.02; $95 \%$ CI 0.90 to $1.16 ; \mathrm{p}=0.71$; moderate certainty of evidence). Visual inspection of the forest plot and $\mathrm{I}^{2}$-statistics $\left(\mathrm{I}^{2}=0 \%\right)$ indicated no heterogeneity. Trial Sequential Analysis showed that we had enough information to reject that ivabradine versus control reduced the risk of myocardial infarction by $15 \%$ or more (RR 1.02; $95 \%$ CI 0.85 to $1.23 ; \mathrm{p}=0.71 ; \mathrm{I}^{2}=0 \% ; \mathrm{D}^{2}=0 \%$ ). This outcome result was assessed at high risk of bias. Incomplete outcome data alone did not seem to have the potential to influence the results. See 'Summary of findingstable' and 'online supplemental 9'.

\section{Non-serious adverse events}

Twenty-four trials randomising 34181 participants reported on non-serious adverse events. Meta-analysis showed evidence of a harmful effect of ivabradine (RR $1.13 ; 95 \%$ CI 1.11 to 1.16 ; $\mathrm{p}<0.00001$; moderate certainty of evidence). Visual inspection of the forest plot and $\mathrm{I}^{2}$-statistics $\left(\mathrm{I}^{2}=8 \%\right)$ indicated low heterogeneity. Trial Sequential Analysis showed that we had enough information to detect that ivabradine versus control increased the risk of non-serious adverse events by $13 \%$ or more (RR $1.13 ; 95 \%$ CI 1.11 to $\left.1.16 ; \mathrm{p}<0.0001 ; \mathrm{I}^{2}=8 \% ; \mathrm{D}^{2}=73 \%\right)$. This outcome result was assessed at high risk of bias. Incomplete outcome data alone did not seem to have the potential to influence the results. See 'Summary of findings-table' and 'online supplemental 10'.

\section{Individual non-serious adverse events}

The trials reported 54 different non-serious adverse events; the SIGNIFY and the BEAUTIFUL trials reported on the majority of those. To limit problems with multiplicity and type 1 errors caused by the unexpected large number of individual serious adverse events, we post-hoc adjusted the threshold for statistical significance to 0.001 when assessing individual non-serious adverse events. ${ }^{10}$

Ivabradine seemed to increase the risk of bradycardia (event classified as 'bradycardia' (RR 4.54; 95\% CI 3.78 to 5.46; $\mathrm{p}<0.0001$; nine trials); event classified as 'heart rate decreased' (RR 8.05; 95\% CI 6.76 to 9.59; p<0.0001; two trials); event classified as 'sinus bradycardia' (RR 9.61; 5.65 to 16.33 ; $\mathrm{p}<0.0001$; one trial)); phosphenes (RR 6.58; $95 \%$ CI 5.34 to 8.10 ; $\mathrm{p}<0.0001$; seven trials); vision blurred (RR 3.39; 95\% CI 2.32 to 4.93; $\mathrm{p}<0.0001$; five trials); QT interval prolonged (RR 2.65; 95\% CI 1.85 to 3.81; $\mathrm{p}<0.0001$; one trial); hypertension (RR 1.19; $95 \%$ CI 1.09 to $1.30 ; \mathrm{p}=0.0001$; four trials); and dizziness ( $R R$ 1.32; $95 \%$ CI 1.13 to $1.54 ; \mathrm{p}=0.0003$; four trials).

Ivabradine seemed to decrease the risk of sinus tachycardia (RR $0.25 ; 95 \%$ CI 0.18 to $0.35 ; \mathrm{p}=0.0001$; one trial).

\section{Exploratory outcomes}

Ivabradine seemed to increase the score of angina frequency (0-100 points, higher score represents a positive outcome; MD 2.06; 95\% CI 0.82 to $3.30 ; \mathrm{p}=0.001$; $\mathrm{I}^{2}=0 \% ; 3$ trials; 4297 participants) and angina stability, both on the Seattle Angina Questionnaire score (0-100 points, higher score represents a positive outcome; MD 1.48; $95 \%$ CI 0.07 to $2.89 ; \mathrm{p}=0.04 ; \mathrm{I}^{2}=0 \% ; 2$ trials; 4217 participants). However, the effect sizes were minimal (SMD 0.1 for angina frequency and SMD 0.06 for angina stability) and both were more than five times below our predefined minimal important difference (SMD 0.5). Furthermore, the SIGNIFY trial accounted for more than $97 \%$ of the total weight in both analyses and used questionable methodology (see the first section of the Results section). We assessed the risk of incomplete outcome data bias to be substantial since $70 \%$ of the participants in the SIGNIFY trial were excluded from the analysis of 
angina, see the first section of the Results section and 'online supplemental 11'.

Four trials assessed exercise tolerance tests. In the ASSOCIATE trial, ivabradine versus control did not reach the minimal important difference in any of the outcome measures in exercise tolerance testing (time to angina onset, time to limiting angina, time to $1 \mathrm{~mm}$ ST depression and total exercise duration). ${ }^{46}$ In the trial by Borer $e t a l,{ }^{28}$ ivabradine treatment reached the minimal important difference for time to angina onset and time to $1 \mathrm{~mm}$ ST depression in the $10 \mathrm{mg}$ ivabradine twice daily group, which is a higher dose than recommended. ${ }^{28} 82$ The participants had discontinued any other anti-ischaemic drug two to 7 days prior to randomisation and the primary outcome was measured after 14 days of treatment. ${ }^{28}$ In two of the unpublished trials, there seemed to be no difference between ivabradine and control in regard to exercise tolerance testing. ${ }^{22} 23$

The results of the remaining exploratory outcomes are reported in the online supplemental material, see 'online supplemental 12' and the Discussion section.

\section{Subgroup analyses}

We post-hoc decided to conduct a subgroup analysis of trials randomising participants with coronary artery disease alone compared to trials randomising participants with both coronary artery disease and heart failure with reduced ejection fraction. We identified 11 trials including participants with both coronary artery disease and heart failure with an ejection fraction of $40 \%$ or less ${ }^{49} 5456-596163646768$ and one trial including participants with an ejection fraction of $45 \%$ or more ${ }^{60}$ We judged trials as being 'coronary artery disease only' trials, if heart failure was not an inclusion criterion, if heart failure was an exclusion criterion, or if there was no mention of heart failure. For serious adverse events, test for subgroup differences showed evidence of a difference $(p<0.00001)$. When analysed separately, we found evidence of a harmful effect of ivabradine in trials randomising participants with coronary artery disease only (RR 1.06; $95 \%$ CI 1.02 to $1.10 ; \mathrm{p}=0.001 ; \mathrm{I}^{2}=27 \% ; 11$ trials) and evidence of a beneficial effect of ivabradine in trials randomising participants with both coronary artery disease and heart failure (RR $0.91 ; 95 \%$ CI 0.86 to $0.96 ; \mathrm{p}=0.0009 ; \mathrm{I}^{2}=0 \%$; two trials). For quality of life, test for subgroup differences showed evidence of a difference $(p<0.00001)$. When analysed separately, we found no evidence of a difference between ivabradine and control in trials randomising participants with coronary artery disease only (SMD -0.05 ; 95\% CI -0.11 to $0.01 ; \mathrm{p}=0.27 ; \mathrm{I}^{2}=17 \%$; two trials) and evidence of a beneficial effect of ivabradine in trials randomising participants with both coronary artery disease and heart failure (SMD $-1.19 ; 95 \% \mathrm{CI}-1.58$ to $-0.81 ; \mathrm{p}<0.00001$; $\mathrm{I}^{2}=99 \%$; two trials).

For serious adverse events, test for subgroup differences showed evidence of a difference $(p<0.0001)$ when comparing trials administering ivabradine at or above median daily dose with trials adminstering ivabradine below median daily dose. When analysed separately, we found evidence of a harmful effect of ivabradine in trials administering ivabradine at or above median daily dose (RR 1.06; 95\% CI 1.02 to $1.10 ; \mathrm{p}=0.001 ; \mathrm{I}^{2}=0 \%$; four trials) and evidence of a beneficial effect of ivabradine in trials administering ivabradine below median daily dose (RR $0.92 ; 95 \%$ CI 0.87 to $0.97 ; p=0.002 ; I^{2}=26 \%$; four trials)

For the remaining subgroup analyses, test for subgroup differences showed no evidence of a difference between ivabradine and control. The results of all subgroup analyses can be found in 'online supplemental 5-7'.

\section{DISCUSSION}

We included a total of 47 randomised clinical trials enrolling 35797 participants. All trials and outcomes were at high risk of bias. ${ }^{21-67}$

Meta-analyses and Trial Sequential Analysis showed that there was no evidence of a difference between ivabradine and placebo or no intervention when assessing all-cause mortality, quality of life, cardiovascular mortality and myocardial infarction.

Meta-analysis showed that ivabradine potentially increased the risk of serious adverse events after removal of outliers from the analyses as well as the following adverse events classified as serious: bradycardia, prolonged QT interval, photopsia, atrial fibrillation and hypertension. Meta-analysis and Trial Sequential Analysis showed that ivabradine seemed to increase the risk of non-serious adverse events.

Ivabradine seemed to increase the score of angina frequency and angina stability on the Seattle Angina Questionnaire. However, we identified several methodological limitations regarding these outcomes (see the Results section). Furthermore, the observed effect sizes seemed minimal as they were more than five times lower than our predefined minimal important difference. ${ }^{14}$

See 'Summary of findings-table' in 'online supplemental 13'.

In a post-hoc subgroup analyses, ivabradine seemed to increase the risk of serious adverse events in trials randomising participants with only coronary artery disease and to decrease the risk of serious adverse events in trials randomising participants with both coronary artery disease and heart failure. Post-hoc analyses need to be interpreted with caution.

Our systematic review has several strengths. Our methodology was predefined and was described in detail in our published protocol. ${ }^{1314}$ We identified 47 trials, whereas the largest of the previous (non-systematic) reviews only included eights trials. ${ }^{83-86}$ To control the risk of random error, we used Trial Sequential Analysis ${ }^{8}$ and adjusted our thresholds for statistical significance. ${ }^{10}$ To control the risk of systematic error, we assessed the risk of bias of all included trials. ${ }^{18} 19$ To assess if the thresholds for statistical and clinical significance were crossed, we used our eight-step procedure. ${ }^{10} \mathrm{We}$ included all randomised clinical trials regardless of publication type, status, language and outcomes. We attempted to 
contact trialists if there were incomplete outcome data or additional information was required.

Our review also has several limitations. All trials were at high risk of bias, including a substantial risk of for-profit bias. ${ }^{80}$ Fourteen of the trials were sponsored by the company that developed ivabradine, including the two largest trials, the SIGNIFY and the BEAUTIFUL trials, that randomised 30019 participants (91\%). ${ }^{21-25} 283032364446545859$ Five of the included trials were unpublished and non-peer reviewed trials of the company that produced ivabradine. ${ }^{21-25}$ Sponsorship of drug trials by manufacturing companies leads to more favourable efficacy results than trials sponsored by other sources. ${ }^{80}$ The BEAUTIFUL and the SIGNIFY trials both used composite outcomes that did not seem to be predefined prior to randomising participants, see the Results section. ${ }^{32}{ }^{5470-73}$ The results of 11 trials were reported only as abstracts, which made the assessment of the methodology and results problematic. ${ }^{27} 293940424547485055$ Therefore, there is a risk that our present results overestimate the beneficial effects and underestimate the harmful effects of ivabradine. ${ }^{8087-92}$ Last, to limit problems with multiplicity and type 1 errors caused by the unexpected large number of individual serious adverse events, we post-hoc adjusted the threshold for statistical significance to 0.001 when assessing individual serious and non-serious adverse events. This threshold was not predefined, and these results should therefore be interpreted with caution. Nevertheless, several of the harmful outcomes may indeed be increased by ivabradine.

Ivabradine was recommended as an effective second-line treatment for angina relief in the 2013 and the 2019 European Society of Cardiology guidelines on the management of chronic coronary artery syndromes. ${ }^{32}$ However, we did not identify any valid evidence supporting that ivabradine should reduce angina symptoms in a clinically significant way. On the contrary, our results show that ivabradine does not seem to have beneficial effects on all-cause mortality, serious adverse events, quality of life, cardiovascular mortality and myocardial infarction. Moreover, ivabradine increases the risk of both serious and non-serious adverse events. The lack of benefit has previously been discussed as being partly due to the decrease in heart rate caused by ivabradine resulting in an increase in systolic blood pressure and left ventricular overload. $^{93}$

\section{CONCLUSION}

Our findings do not support that ivabradine offers significant benefits on patient important outcomes, but rather seems to increase the risk of serious adverse events such as atrial fibrillation and non-serious adverse events. Based on current evidence, guidelines need reassessment and the use of ivabradine for angina pectoris should be reconsidered.

\section{Differences between protocol and review}

We conducted our literature search in parallel with another review on the effects of adding ivabradine to usual care in participants with heart failure. We originally planned to report and analyse the results including participants with angina and participants with heart failure in one review, but due to clinical and statistical heterogeneity and reviewer recommendations, we decided to report the results in two separate reviews. ${ }^{14}$

\section{Author affiliations}

${ }^{1}$ Copenhagen Trial Unit, Centre for Clinical Intervention Research, Copenhagen University Hospital, Copenhagen, Denmark

${ }^{2}$ Department of Internal Medicine, Holbæk Hospital, Holbæk, Denmark ${ }^{3}$ Institute of Basic Research in Clinical Medicine, China Academy of Chinese Medical Sciences Guanganmen Hospital, Xicheng District, China

${ }^{4}$ Centre for Evidence-Based Chinese Medicine, Beijing University of Chinese Medicine, Beijing, China

${ }^{5}$ The Cochrane Hepato-Biliary Group, Copenhagen Trial Unit, Centre for Clinical Intervention Research, Copenhagen University Hospital, Copenhagen, Denmark ${ }^{6}$ Department of Regional Health Research, The Faculty of Health Sciences, University of Southern Denmark, Odense, Denmark

Acknowledgements The authors would like to thank Liliya Ziganshina for assisting them with screening and data extracting articles in Russian.

Contributors MM: conceived the systematic review, conducted literature search, data extraction, data analysis, data interpretation and wrote the article. EEN, LN and S-hY: conducted literature search, data extraction and amended the article. NJS: conducted data extraction and amended the article. CG: helped conceive the systematic review, provided invaluable comments and amended the article. JCJ: conceived the systematic review, aided in data interpretation and amended the article.

Funding Funding by the Copenhagen Trial Unit by wages paid to JCJ and CG. The funding is through the Danish Finance Act.

Disclaimer The funding source had no influence on the systematic review.

Competing interests None declared.

Patient consent for publication Not required.

Provenance and peer review Not commissioned; externally peer reviewed.

Data availability statement Data are available upon reasonable request. All data relevant to the study are included in the article or uploaded as supplementary information. All data are available as supplementary files. Data can also be requested from the corresponding author.

Open access This is an open access article distributed in accordance with the Creative Commons Attribution Non Commercial (CC BY-NC 4.0) license, which permits others to distribute, remix, adapt, build upon this work non-commercially, and license their derivative works on different terms, provided the original work is properly cited, appropriate credit is given, any changes made indicated, and the use is non-commercial. See: http://creativecommons.org/licenses/by-nc/4.0/.

\section{ORCID iDs}

Mathias Maagaard http://orcid.org/0000-0002-9037-7295

Emil Eik Nielsen http://orcid.org/0000-0003-1108-9533

\section{REFERENCES}

1 GBD 2013 Mortality and Causes of Death Collaborators. Global, regional, and national age-sex specific all-cause and causespecific mortality for 240 causes of death, 1990-2013: a systematic analysis for the global burden of disease study 2013. Lancet 2015;385:117-71.

2 Roth GA, Johnson C, Abajobir A, et al. Global, regional, and national burden of cardiovascular diseases for 10 causes, 1990 to 2015. J Am Coll Cardiol 2017;70:1-25.

3 Task Force Members, Montalescot G, Sechtem U, et al. 2013 ESC guidelines on the management of stable coronary artery disease: the task force on the management of stable coronary artery disease of the European Society of cardiology. Eur Heart J 2013;34:2949-3003.

4 Weeda ER, Nguyen E, White CM. Role of ivabradine in the treatment of patients with cardiovascular disease. Ann Pharmacother 2016;50:475-85.

5 Sulfi S, Timmis AD. Ivabradine -- the first selective sinus node I(f) channel inhibitor in the treatment of stable angina. Int $\mathrm{J}$ Clin Pract 2006;60:222-8

6 López-Bescós L, Filipova S, Martos R. Long-term safety and efficacy of ivabradine in patients with chronic stable angina. Cardiology 2007;108:387-96. 
7 Tse S, Mazzola N, Ivabradine MN. Ivabradine (Corlanor) for heart failure: the first selective and specific I F inhibitor. Pharm Ther $2015 ; 40: 810-4$.

8 Wetterslev J, Jakobsen JC, Gluud C. Trial sequential analysis in systematic reviews with meta-analysis. BMC Med Res Methodol 2017:17:39.

9 Keus F, Wetterslev J, Gluud C, et al. Evidence at a glance: error matrix approach for overviewing available evidence. BMC Med Res Methodol 2010;10:90.

10 Jakobsen JC, Wetterslev J, Winkel P, et al. Thresholds for statistical and clinical significance in systematic reviews with meta-analytic methods. BMC Med Res Methodol 2014;14:120.

11 Higgins JPT, Thompson SG. Quantifying heterogeneity in a metaanalysis. Stat Med 2002;21:1539-58.

12 Moher D, Liberati A, Tetzlaff J, et al. Preferred reporting items for systematic reviews and meta-analyses: the PRISMA statement. BMJ 2009;339:b2535

13 Maagaard M, Nielsen EE, Gluud C, et al. Ivabradine for coronary artery disease and/or heart failure: a protocol for a systematic review of randomised clinical trials with meta-analysis and trial sequential analysis. PROSPERO 2018 CRD42018112082. Available: http://www.crd.york.ac.uk/PROSPERO/display_record.php?ID= CRD42018112082

14 Maagaard M, Nielsen EE, Gluud C, et al. Ivabradine for coronary artery disease and/or heart failure-a protocol for a systematic review of randomised clinical trials with meta-analysis and trial sequential analysis. Syst Rev 2019;8:39.

15 Higgins JP, Green S. Cochrane Handbook for systematic reviews of interventions, 2011. Available: www.cochrane-handbook.org

16 The Cochrane Collaboration. Review Manager (RevMan) [program] 5.3 version. Copenhagen: The Nordic Cochrane Centre, 2014. https://training.cochrane.org/online-learning/core-softwarecochrane-reviews/revman/revman-5-download

17 Thorlund K, Devereaux PJ, Wetterslev J, et al. Can trial sequential monitoring boundaries reduce spurious inferences from metaanalyses? Int J Epidemiol 2009;38:276-86.

18 Schünemann H, Brożek J, Guyatt G, et al. GRADE Handbook for grading quality of evidence and strength of recommendations. The GRADE Working Group, 2013. https://gdt.gradepro.org/app/ handbook/handbook.html

19 Guyatt GH, Oxman AD, Vist GE, et al. GRADE: an emerging consensus on rating quality of evidence and strength of recommendations. BMJ 2008;336:924-6.

20 Servier. ClinicalTrial Servier, 2019. Available: https://clinicaltrials. servier.com/ [Accessed 08 Dec 2019].

21 Servier. Evaluation versus placebo of the effects on heart rate, haemodynamic parameters, safety and tolerability of $5 \mathrm{mg}$ bolus of ivabradine followed by 8 -hour infusion of $5 \mathrm{mg}$ of ivabradine, given to patients undergoing a percutaneous coronary intervention following a myocardial infarction with ST segment elevation (STEMI). A pilot, blind, randomised, placebo-controlled, international multicentre trial. Including the ancillary MRI sub-study. CL2-16257060, 2009. Available: https://clinicaltrials.servier.com/find-clinicaltrials/

22 Servier. Evaluation of the anti-anginal efficacy and safety of ivabradine used in combination with an anti-anginal monotherapy in patients with stable effort angina pectoris. A 6-week, randomised, double-blind controlled, parallel-group, international, multicentre study. CL3-16257-064, 2010. Available: https://clinicaltrials.servier. $\mathrm{com} /$ find-clinical-trials/

23 Servier. Evaluation of the anti-anginal efficacy and safety of oral administration of ivabradine compared to placebo on top of a background therapy with a calcium antagonist (amlodipine or nifedipine) in patients with stable angina pectoris. A 6-week randomised double-blind parallel-group international multicentre study. CL3-16257-068, 2012. Available: https://clinicaltrials.servier. com/find-clinical-trials/

24 Servier. Effects of oral chronic administration of ivabradine $(7.5 \mathrm{mg}$ b.i.d.) in comparison to placebo (b.i.d.) on top of beta-blockers, on central aortic blood pressure. Randomised, cross-over, double-blind, multicentre, study over 10 weeks in patients with stable coronary artery disease and a resting heart rate equal or superior to $70 \mathrm{bpm}$, already treated with beta-blockers. CL2-16257-096, 2014. Available: https://clinicaltrials.servier.com/find-clinical-trials/

25 Servier. Long-term (3 years) ophthalmic safety and cardiac efficacy and safety of ivabradine administered orally at the therapeutic doses (2.5/5/7.5 mg b.i.d.) on top of anti-anginal background therapy, to patients with chronic stable angina pectoris. An international, double-blind placebo controlled study. CL3-16257-067, 2015. Available: https://clinicaltrials.servier.com/find-clinical-trials/
26 Adel M, Mansour S, Sabri NA, et al. A clinical study evaluating the effect of ivabradine on non ST-segment elevation acute coronary syndromes. Int J Pharmaceut Sci Res 2016;7:1441-9.

27 Ageev FT, Makarova GV, Patrusheva IF, et al. [The efficacy and safety of the combination of $\beta$-blocker bisoprolol and if inhibitor I(f) ivabradine in patients with stable angina and chronic obstructive pulmonary disease]. Kardiologiia 2010;50:22-6.

28 Borer JS, Fox K, Jaillon P, et al. Antianginal and antiischemic effects of ivabradine, an I(f) inhibitor, in stable angina: a randomized, double-blind, multicentered, placebo-controlled trial. Circulation 2003;107:817-23.

29 Cay S, Cagirci G, Sen N, et al. Ivabradine improves short-term outcomes after primary percutaneous coronary intervention in patients presenting with acute ST-segment elevation myocardial infarction. Eur Heart J 2011;32:868.

30 Dillinger J-G, Maher V, Vitale C, et al. Impact of ivabradine on central aortic blood pressure and myocardial perfusion in patients with stable coronary artery disease. Hypertension 2015;66:1138-44.

31 Dominguez-Rodriguez A, Consuegra-Sanchez L, Blanco-Palacios G, et al. Anti-inflammatory effects of ivabradine in patients with acute coronary syndrome: a pilot study. Int J Cardiol 2012;158:160-2.

32 Fox K, Ford I, Steg PG, et al. Ivabradine in stable coronary artery disease without clinical heart failure. N Engl J Med 2014;371:1091-9.

$33 \mathrm{Gao} H$, Wei XH, Han GX. Clinical observation of ivabradine combined with amlodipine in treatment of chronic stable angina pectoris. Drug Clin 2017;32:1854-8.

34 Gloekler S, Traupe T, Stoller M, et al. The effect of heart rate reduction by ivabradine on collateral function in patients with chronic stable coronary artery disease. Heart 2014;100:160-6.

$35 \mathrm{Hao} \mathrm{J}, \mathrm{XL}$ L, CM Q, et al. Clinical observation of effect of ivabradine in the treatment of patients with chronic obstructive pulmonary disease complicated with angina pectoris. Chin J Cardiovasc Med 2016;21:475-7.

36 Hohneck AL, Fries P, Ströder J, et al. Effects of heart rate reduction with ivabradine on vascular stiffness and endothelial function in chronic stable coronary artery disease. $J$ Hypertens 2019;37:1023-31.

37 Huang J, Qian HY, ZC H, et al. The effect of ivabradine on the leve Is of cystatin $C$ in elderly patients with chronic heart failure. Chin $J$ Difficult Complex cases 2017;16:545-9.

38 Huang YP. Analysis of the effect of ivabradine combined with bisoprolol on coronary heart disease after PCl. Strait Pharmaceut J 2018;30:177-8.

39 Kadro W. The effect of ivabradine on silent ambulatory myocardial ischemia. J Hypertension 2015;33:e30.

40 Lamendola P, Di Franco A, Di Monaco A, et al. Effect of heart rate reduction on peripheral and coronary endothelial function in patients with microvascular angina. Giornale Italiano di Cardiologia 2011;12:e52.

41 Mangiacapra F, Colaiori I, Ricottini E, et al. Heart rate reduction by ivabradine for improvement of endhotelial function in patients with coronary artery disease: the randomized open-label RIVENDEL study. Eur Heart J 2015;36:1105.

42 Naji F, Kanic V. Pretreatment with ivabradine reduces periprocedural myocardial injury and infarction in stable ischemic patients undergoing coronary intervention. Eur Heart J 2014;35:127-8.

43 Shavarov AA, Kobalava ZD, Kiyakbaev GK, et al. [Clinical efficacy and safety of early administration of ivabradine in patients with acute coronary syndrome]. Kardiologiia 2015;55:54-62.

44 Steg PG, Lopez-de-Sà E, Schiele F, et al. Safety of intravenous ivabradine in acute ST-segment elevation myocardial infarction patients treated with primary percutaneous coronary intervention: a randomized, placebo-controlled, double-blind, pilot study. Eur Heart J Acute Cardiovasc Care 2013;2:270-9.

45 Taccheri T, Calcagno S, Carnesale R, et al. Comparison of the efficacy and tolerability of ivabradine vs ranolazine vs standard medical therapy in patients of chronic stable angina pectoris despite coronary percutaneous revascularization. Eur Heart J 2014;35:323.

46 Tardif J-C, Ponikowski P, Kahan T, et al. Efficacy of the I(f) current inhibitor ivabradine in patients with chronic stable angina receiving beta-blocker therapy: a 4-month, randomized, placebo-controlled trial. Eur Heart J 2009:30:540-8.

47 Vasyuk YA, Shkolnik E, Lebedev EV. Ivabradine in the treatment of stable angina early after myocardial infarction. Eur $\mathrm{J}$ Heart Failure 2009;8.

48 Vatinian S, Gurgenyan S, Nikoghosyan K. Effectiveness of combination therapy using nebivolol and ivabradine in patients with essential hypertension and stable effort angina. Eur Heart $J$ 2013;34:722

49 Vatinian S, Gurgenyan S, Nikoghosyan K. Efficacy of addition of ivabradine to bisoprolol in patients with essential hypertension, 
coronary artery disease and reduced left ventricular systolic function. Eur Heart J 2015;36:672-3.

50 Villano A, Di Franco A, Nerla R, et al. Effects of ranolazine and ivabradine on exercise stress test and on coronary and peripheral vascular function in patients with refractory microvascular angina. Eur Heart J 2012;33:780.

51 Wang K, XY Q, TT L. Clinical efficacy evaluation of ivabradine in treatment of patients with stable angina pectoris complicated with chronic obstructive pulmonary disease. Chin J New Drugs Clinical Rem 2018;37:53-7.

52 Zhang Y, Li D, Liu Q, et al. Effect of ivabradine tablets on left ventricular function in patients with coronary heart disease and angina pectoris. China Pharmaceut 2018;27:58-60.

53 Zhao HZ. Ivabradine hydrochloride on the Efficacy and safety of chronic stable angina pectoris. World Latest Med Inform 2017;17:52-3.

54 Fox K, Ford I, Steg PG, et al. Ivabradine for patients with stable coronary artery disease and left-ventricular systolic dysfunction (beautiful): a randomised, double-blind, placebo-controlled trial. Lancet 2008;372:807-16.

55 Tagliamonte E, Alfano D, Astarita R, et al. Combined effect of ivabradine on coronary flow reserve and left ventricular contractile reserve in patients with coronary microvascular disease. Eur Heart $J$ Cardiovasc Imag 2019;20.

56 Barillà F, Pannarale G, Torromeo C, et al. Ivabradine in patients with ST-elevation myocardial infarction complicated by cardiogenic shock: a preliminary randomized prospective study. Clin Drug Investig 2016;36:849-56.

$57 \mathrm{Hu}$ SD, Cao MQ, XB L, et al. Efficacy of ivabradine in treating acute myocardial infarction patients with heart failure. Jiangsu Med 2018:44:1428-31.

58 Nguyen L, Squara P, Amour J, et al. Intravenous ivabradine in low cardiac output syndrome after cardiac surgery treated by dobutamine: a phase II trial. Arch Cardiovasc Dis Supp/ 2018;10:41.

59 Sallam M, Al-Saadi T, Alshekaili L, et al. Impact of ivabradine on health-related quality of life of patients with ischaemic chronic heart failure. Curr Vasc Pharmacol 2016;14:481-6.

60 Tatarchenko IP, Pozdniakova NV, Biriuchenko MV, et al. [Clinical efficacy of ivabradin and nebivolol addition in combined treatment of ischemic heart disease patients with left ventricular dysfunction]. Ter Arkh 2008;80:40-4.

61 Zhang JH, Sun CF. Clinical effect of ivabradine on coronary heart disease combined with chronic obstructive pulmonary disease. Shaanxi Med J 2019;48:256-8.

62 Chen TH, Liu KD, Tan LY. Effect of ivabradine combined with bisoprolol on cardiac rehabilitation in patients with coronary heart disease after percutaneous coronary intervention. China Med Pharm 2019;9:36-8

$63 \mathrm{He} \mathrm{HT}$, GH K, Fang YF. The improvement of ventricular remodeling in patients with coronary heart disease and heart failure by ivabradine. Contemp Med 2019;25:70-3.

64 Wang WQ. Effect of ivabradine and nicorandil on cardiac functions and exercise endurance in patients with coronary heart disease after PCl. Chin J Rational Drug Use 2019;16:111-3.

65 Zhou YN. Effect of ivabradine on patients with coronary atherosclerotic heart disease and angina pectoris and influence on left ventricular function. Chin J Clin Rational Drug Use 2019;12:22-3.

66 Di T, Cao HT, Wang Y, et al. Effect of ivabradine on patients with diabetes mellitus with coronary heart disease. Chin J Cardiovasc Res 2020;18:146-50

67 Zhang ZC. Effect of ivabradine combined with metoprolol on cardiac function and carotid intima-media thickness of patients with coronary heart disease complicated with heart failure. Med J Chin People Health 2020;32:11-13.

68 Sayganov SA, Grishkin YN. Selective I-f-channel inhibitor ivabradine in the treatment of sinus tachycardia in coronary heart disease patients with acute heart failure and inferior myocardial infarction. Cardiovasc Ther Prevent 2010;9:52-6.

69 Huang W, Yu J, Liu M, et al. The effect of ivabradine combined with bisoprolol on rehabilitation of patients with coronar y heart disease after p ercutaneous coronar y intervention. Chin Hosp Pharm J 2017:37:151-4.

70 Fox K, Ferrari R, Tendera M, et al. Rationale and design of a randomized, double-blind, placebo-controlled trial of ivabradine in patients with stable coronary artery disease and left ventricular systolic dysfunction: the morBidity-mortality EvAlUaTion of the I(f) inhibitor ivabradine in patients with coronary disease and left ventricULar dysfunction (BEAUTIFUL) study. Am Heart J 2006;152:860-6.
71 Servier. The beautiful study: effects of ivabradine in patients with stable coronary artery disease and left ventricular systolic dysfunction. ClinicalTrials.gov: Servier, 2005.

72 Fox K, Ford I, Steg PG, et al. Rationale, design, and baseline characteristics of the study assessing the morbidity-mortality beNefits of the if inhibitor ivabradine in patients with coronarY artery disease (signify trial): a randomized, double-blind, placebocontrolled trial of ivabradine in patients with stable coronarY artery disease without clinical heart failure. Am Heart J 2013;166:654-61.

73 Servier. Effects of ivabradine in patients with stable coronary artery disease without clinical heart failure (SIGNIFY). ClinicalTrials.gov: Servier, 2015

74 Tendera M, Chassany O, Ferrari R, et al. Quality of life with ivabradine in patients with angina pectoris: the study assessing the Morbidity-Mortality benefits of the if inhibitor ivabradine in patients with coronary artery disease quality of life substudy. Circ Cardiovasc Qual Outcomes 2016:9:31-8.

75 Hróbjartsson A, Emanuelsson F, Skou Thomsen AS, et al. Bias due to lack of patient blinding in clinical trials. A systematic review of trials randomizing patients to blind and nonblind sub-studies. Int $J$ Epidemiol 2014;43:1272-83.

76 Hróbjartsson A, Thomsen ASS, Emanuelsson F, et al. Observer bias in randomised clinical trials with binary outcomes: systematic review of trials with both blinded and non-blinded outcome assessors. BMJ 2012;344:e1119.

77 Hróbjartsson A, Thomsen ASS, Emanuelsson F, et al. Observer bias in randomized clinical trials with measurement scale outcomes: a systematic review of trials with both blinded and nonblinded assessors. CMAJ 2013;185:E201-11.

78 Savović J, Jones HE, Altman DG, et al. Influence of reported study design characteristics on intervention effect estimates from randomized, controlled trials. Ann Intern Med 2012;157:429-38.

79 Savović J, Turner RM, Mawdsley D, et al. Association between Riskof-Bias assessments and results of randomized trials in Cochrane reviews: the ROBES Meta-Epidemiologic study. Am J Epidemiol 2018;187:1113-22.

80 Lundh A, Lexchin J, Mintzes B, et al. Industry sponsorship and research outcome. Cochrane Database Syst Rev 2017;29.

81 Thorlund K, Engstrøm J, Wetterslev J, et al. User manual for Trial Sequential Analysis (TSA): Copenhagen Trial Unit, 2017. Available: http://www.ctu.dk/tsa/files/tsa_manual.pdf [Accessed 10 Dec 2019].

82 Knuuti J, Wijns W, Saraste A, et al. 2019 ESC guidelines for the diagnosis and management of chronic coronary syndromes. Eur Heart J 2020;41:407-77.

83 Ye L, Ke D, Chen Q, et al. Effectiveness of ivabradine in treating stable angina pectoris. Medicine 2016;95:e3245.

84 Tanboğa İbrahim Halil, Topçu S, Aksakal E, et al. The risk of atrial fibrillation with ivabradine treatment: a meta-analysis with trial sequential analysis of more than 40000 patients. Clin Cardiol 2016;39:615-20.

85 Kang S, Li C-J, Zhang X-M. Ivabradine has a neutral effect on mortality in randomized controlled trials. Medicine 2017;96:e8067.

86 Cammarano C, Silva M, Comee M, et al. Meta-analysis of ivabradine in patients with stable coronary artery disease with and without left ventricular dysfunction. Clin Ther 2016:38:387-95.

87 Wood L, Egger M, Gluud LL, et al. Empirical evidence of bias in treatment effect estimates in controlled trials with different interventions and outcomes: meta-epidemiological study. BMJ 2008;336:601-5

88 Schulz KF, Chalmers I, Hayes RJ, et al. Empirical evidence of bias. Dimensions of methodological quality associated with estimates of treatment effects in controlled trials. JAMA 1995;273:408-12.

89 Savović J, Jones $\mathrm{H}$, Altman D, et al. Influence of reported study design characteristics on intervention effect estimates from randomised controlled trials: combined analysis of metaepidemiological studies. Health Technol Assess 2012;16:1-82.

90 Moher D, Pham B, Jones A, et al. Does quality of reports of randomised trials affect estimates of intervention efficacy reported in meta-analyses? Lancet 1998;352:609-13.

91 Kjaergard LL, Villumsen J, Gluud C. Reported methodologic quality and discrepancies between large and small randomized trials in meta-analyses. Ann Intern Med 2001;135:982-9.

92 Gluud LL. Bias in clinical intervention research. Am J Epidemio 2006;163:493-501

93 Messerli FH, Rimoldi SF, Bangalore S, et al. When an Increase in Central Systolic Pressure Overrides the Benefits of Heart Rate Lowering. J Am Coll Cardiol 2016;68:754-62. 